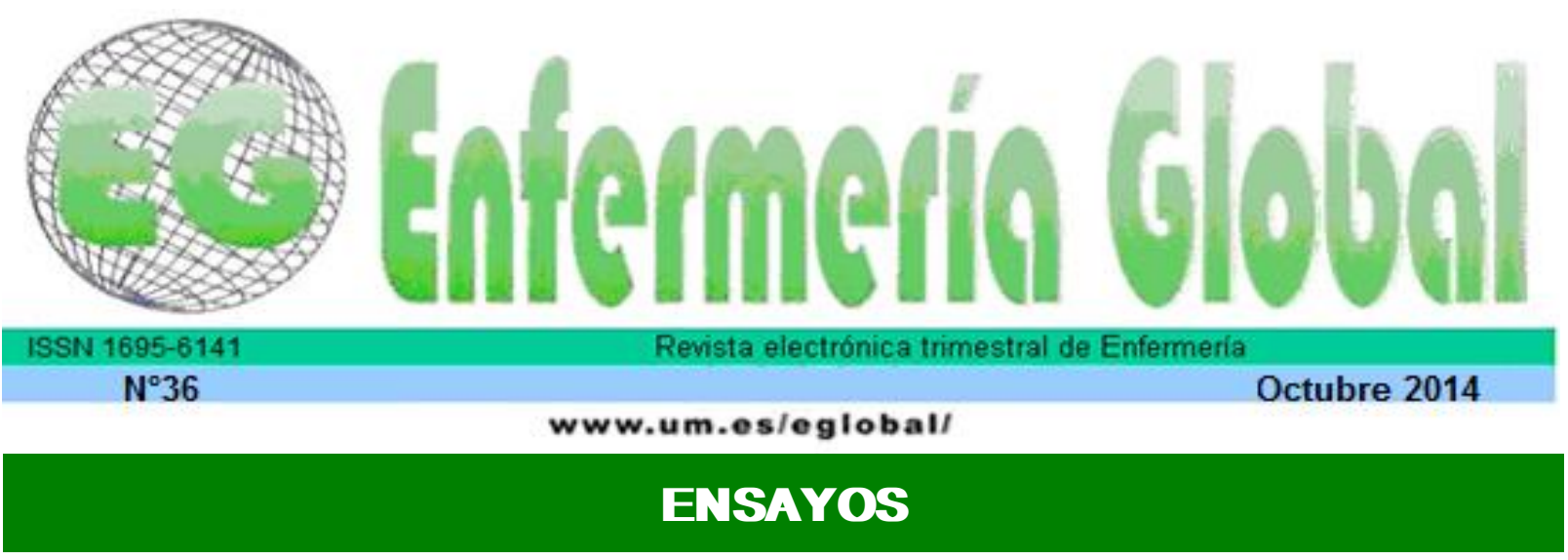

\title{
Del cuidado previsible al cuidado complejo de enfermería
}

Do cuidado previsível ao cuidado complexo de enfermagem

From predictable care to complex nursing care

\section{*Backes, Dirce Stein *Zamberlan, Claudia *Freitas, Hilda Barbosa de ${ }^{*}$ Colomé, Juliana *Souza, Martha Teixeira de *Costenaro, Regina Santini}

\author{
*Doctora en Enfermería. Docente del Curso de Enfermería del Centro Universitario Franciscano - \\ UNIFRA.Santa Maria. Brasil. E-mail: backesdirce@ig.com.br
}

\section{Palabras clave: Cuidados en Enfermería; Dinámica no Lineal; Teoría de sistemas Palavras chave: Cuidados de enfermagem; Dinâmica não linear; Teoria de sistemas} Keywords: Nursing Care; Nonlinear Dynamics; Systems Theory

\section{RESUMEN}

El estudio tiene como objetivo presentar un ensayo teórico que permite ampliar el cuidado de enfermería como un fenómeno complejo, en el cual y para el cual, a menudo, no se encuentran respuestas lineales y previsibles. Se señalan aspectos que impulsan la necesidad de adoptar nuevos modelos de pensamiento, capaces de manejar los procesos negativos y contradictorios en la vida cotidiana del ser y del hacer de la enfermería. Se trata, en resumen de la necesidad de superar la lógica de la fragmentación, de la previsibilidad, de la seguridad y el orden excesivo -de las recetas de atención - todavía presentes en las prácticas de cuidado.

\section{RESUMO}

Objetiva-se apresentar um ensaio reflexivo que possibilita ampliar o cuidado de enfermagem como fenômeno complexo, no qual e para o qual, frequentemente, não se tem respostas lineares e previsíveis. Sinalizam-se aspectos que direcionam para a necessidade de adotar novos modelos de pensamento, capazes de comportar os processos adversos e contraditórios no dia a dia do ser e fazer enfermagem. Discute-se, em suma, a necessidade de superar a lógica da fragmentação, da previsibilidade, da certeza e da excessiva ordem - receitas prontas de cuidado - ainda marcante nas práticas de cuidado.

\section{ABSTRACT}

This study aims to present a theoretical reflection that enables to expand nursing care as a complex phenomenon, in which and for which, frequently, are not have linear and predictable answers. Signal up aspects that drive the need to adopt new models of thought, capable of admit the adverse and contradictory processes in everyday life of being and doing nursing. It discusses, need to overcome the 
logic of fragmentation, of predictability, of certainty and of the excessive order - ready recipes of care still outstanding in care practices.

\section{CONSIDERACIONES INICIALES}

El proceso de cuidar/cuidado y la formación académica de los profesionales de enfermería ha sido, a lo largo de los años, uno de los principales objetos de reflexión y de investigación. En la búsqueda de la comprensión ampliada y sistémica de la atención de salud, así como la búsqueda de nuevas posibilidades para intervenir en la dinámica de la vida saludable, entendida como un proceso singular, circular e interactivo, dinamizado por medio de las experiencias de orden y desorden ${ }^{(1)}$, el enfermero está convirtiéndose en uno de los principales protagonistas.

Al concebir la enfermería como ciencia, arte o tecnología de promoción de los cuidados de enfermería al ser humano en su singularidad y multidimensionalidad, articulada con los demás profesionales comprometidos con el fenómeno salud ${ }^{(2)}$, se supone que sólo un concepto ampliado de cuidar/cuidado es capaz de darse cuenta de la complejidad del ser - ser en un continuo proceso de auto-organización para una vida mejor - una vida saludable.

Una encuesta reciente de los conceptos y las connotaciones de la atención de enfermería muestra que ha adquirido, crecientemente, los contornos ontológicos y epistemológicos que contemplan los conceptos de atención como la esencia del ser humano; como proceso interactivo y asociativo; ambiente de cuidado; sistema de atención; proceso relacional entre individuos, familias y comunidades, y como un bien social que promueve la vida y el vivir más saludable ${ }^{(3-11)}$.

Con base en las conclusiones anteriores y en nuevos estudios sobre la temática, se argumenta que la atención de enfermería es un fenómeno complejo, dinámico, interrelacional y sistematizado, a través de múltiples relaciones, interacciones y asociaciones sistémicas, con el fin de promover y restaurar la salud del ser humano en su totalidad y articulada con todo a su alrededor ${ }^{(12)}$. Se reconoce, sin embargo, que el cuidar/cuidado de enfermería redujo/reduce, a menudo, a una acción puntual, lineal y fragmentada, o sea, a una acción vertical y determinista entre sujeto - objeto.

Se concibe que la ciencia clásica disolviera la complejidad de los fenómenos para revelar la simplicidad oculta de las leyes de la naturaleza. Este comportamiento se justificaba por el hecho de que la mejor hipótesis era más simple o la que apelaba para un número más reducido de entidades explicativas. El autor llama la atención sobre el hecho de que la ciencia tiene necesidades no sólo de un conocimiento apto a considerar la complejidad de lo real, sino para transformar este conocimiento de complejidad en pensamiento sistemático ${ }^{(13)}$.

El término complejo, al cual nos referimos aquí, por lo general evoca diversas expresiones, como el cuidado es complejo, la situación es compleja, la búsqueda de soluciones es una tarea compleja. Algo es complejo también cuando por lo menos envuelve más de una circunstancia y/o posibilidad interactiva, es decir, tiene una multitud de interacciones, asociaciones e interfaces establecidas entre un gran número de unidades, expresadas por las diferentes dimensiones del cuidado y salud $^{(14-15)}$.

Al comprender la complejidad del cuidar/cuidado de enfermería en las diferentes situaciones y, asimismo, la necesidad de avanzar en los marcos teóricos que amplían 
el campo de visión del enfermero, que tiene como objetivo presentar un ensayo teórico que permite ampliar el cuidar/cuidado de enfermería como un fenómeno complejo, en el cual y por el cual, a menudo, no se encuentran respuestas lineales y predecibles.

\section{EXPLICITANDO LAS RAZONES PARA LA CONSTRUCCIÓN DE ESTE ESTUDIO}

Se trata de un ensayo teórico, de carácter reflexivo, tiene como objetivo contribuir a la expansión del cuidar/cuidado de enfermería como un fenómeno complejo. Este objetivo busca trascender la concepción puntual, lineal y predecible del cuidado aún fuertemente presente en el pensar y actuar de los profesionales de enfermería. El estudio está relacionado a un proyecto de investigación titulado "Cuidado de Enfermería Complejo", aprobado por el Comité de Ética en Investigación, bajo el $173 / 2011$.

La motivación para la construcción de este estudio, por lo tanto, se originó en los informes, expresados en diferentes áreas académicas de la convivencia académica, como el aula, campo de prácticas, grupos de estudio y reflexión entre investigadores, así como observaciones en diferentes escenarios de práctica. En medio de los distintos debates, a menudo, eran comunes los informes, provenientes de alumnos, profesores o enfermeros asistenciales, lo que permitió reflexiones y nuevas preguntas, sobre todo, en los grupos de estudio y de investigación entre los investigadores. Para ejemplificar tales investigaciones, se relatan las tres afirmaciones siguientes (D1, D2, D3), y seleccionadas aleatoriamente y con el debido permiso de los entrevistados, alos cuales servirán de fuente de inspiración para la discusión reflexiva del cuidar/cuidado de enfermería en la perspectiva de la complejidad.

"Sabes, esta noche al acostarme me quedé pensando mucho en aquella paciente, en fase terminal, y que en varias ocasiones me interrogó ayer: ¿'Me voy a morir pronto'? Lo confieso, me quedé sin respuesta, sin saber lo que decir y lo que hacer. Me sentí muy mal... La pregunta de aquella señora aún continua en mi pensamiento"(D1).

"Cuando empeoró su cuadro, el médico pidió su traslado a la Unidad de Cuidados Intensivos Pediátricos. Al entrar en la habitación para moverlo, el niño de seis años de pronto me dijo: 'Yo no quiero ir a la UCl'. Entonces le expliqué, que allí estaría mejor y tendría profesionales para cuidarle con mayor intensidad. El niño, sin embargo, dispensó los comentarios y de inmediato intervino con el siguiente comentario: 'Yo no quiero ir a la UCl, allí voy quedarme sin mis juguetes, sin mis cositas... Quiero quedarme aquí en mi habitación con mis amigos'. ¡Dios mío! Me quedé sin respuesta. No podía pensar en otra cosa"(D2).

"Estaba entrando en el plantón, el familiar de una habitación me pidió ayuda. El paciente, en fase terminal, presentaba hemorragia intensa. La sangre se derramaba por todos los orificios. Al localizar al médico, él solicitó que se le prestaran solamente los cuidados paliativos y que no tenía nada más que hacer. Al ver la hemorragia incontrolable y la desesperación de su esposa, no sabía que pensar, que decirle y ni lo que hacer en el momento. Me sentí completamente impotente, indefensa... Empecé a orar..." (D3). 


\section{ME QUEDÉ SIN RESPUESTA, SIN SABER LO QUE PENSAR, DECIR O HACER... ELEMENTOS PARA REFLEXIÓN}

La expresión "me quedé sin respuesta, sin saber lo que pensar, lo que decir y lo que hacer" se hizo evidente en las declaraciones anteriores, pero también son muy comunes en la práctica de enfermería, en diferentes espacios. Por un lado, el término carga un sentimiento de impotencia y expresa la vulnerabilidad del profesional de enfermería ante las adversidades, a los que no tienen respuestas, por otro lado, evidencia la concepción lineal, puntual y reduccionista del cuidar/cuidado, como reflejo de un saber cartesiano, mecanicista y fragmentado.

El hombre a través de su conciencia, su capacidad de proyectar el futuro, de innovar y crear adquirió progresivamente poder para influir en los demás y cambiar el curso de los acontecimientos específicamente en los dominios más profundas de la vida. Por otro lado, un mundo donde domina la innovación es un mundo incierto. En analogía con lo anterior se corrobora que el cuidado/cuidar de enfermería a pesar de innovador y dinámico, a menudo, no encuentra respuestas para la modificación del curso de los acontecimientos y de los dominios de la vida, esto porque vivir es impredecible y toda la noción de equilibrio en movimiento está en la lógica de la vida ${ }^{(16)}$.

Se observa, comúnmente, que el profesional de enfermería tiene la necesidad de encontrar y dar respuestas a las diferentes preguntas y/o situaciones de la vida cotidiana. Cuando no puede encontrarlas en sí o en el otro, a veces, se siente frustrado, aislado, deprimido y/o se considera inferior, incapaz o incompetente profesionalmente. Tales reacciones son evidentes en la medida que los profesionales relatan que no consiguieron dormir por la noche, no consiguieron dejar de pensar en las preguntas y/o situación adversa, entre otros.

Podríamos preguntarnos: ¿por qué necesitamos, despreciar, encontrar respuestas a las diferentes preguntas y/o situaciones de la vida cotidiana, si comprendemos que el ser humano es un ser singular y complejo, para el que necesitamos respuestas igualmente complejas? ¿Por qué nos sentimos impotentes, incapaces o inseguros ante la enfermedad terminal de la vida, a pesar de su finitud? En fin, ¿por qué necesitamos certezas absolutas o recetas listas de cuidado, sabiendo que la creatividad surge de las posibilidades interactivas y de situaciones aparentemente adversas y contradictorias?

Para comprender esta tarea, es necesario analizar, a priori, cuál es el modelo de pensamiento que direccionó o está direccionando el cuidar/cuidado de enfermería. La enfermería, como los demás conocimientos del área de la salud, sigue estando aún regida por el modelo reduccionista cartesiano, pautado por sistemas simples, lineales, predecibles, controlables y constituidos de partes separadas, es decir, divisible y fragmentado en partes. En esta relación, el todo es sólo la suma de las partes ${ }^{(17-18)}$. Este modelo de sistema fue y en muchos casos sigue siendo, interpretado por profesionales de enfermería en su intento de encontrar certezas absolutas y previsibles para las diferentes realidades del cuidado.

Se admite, sin embargo, que el modelo de pensamiento fragmentado y reduccionista ha sido superado, aún incipientemente, por marcos sistémicos, no lineales e impredecibles, capaces de provocar el pensamiento humano, con el fin de aumentar la percepción de los fenómenos sociales. Una de estas referencias se refiere al pensamiento complejo como se indica por Morin ${ }^{(19)}$. 
Nacido en Francia, en 1921, Edgar Morin es reconocido como uno de los pensadores más influyentes en la búsqueda de la sistematización de una epistemología de la complejidad. El autor señala la necesidad de una reforma del pensamiento favorable a la reconexión del conocimiento. Destaca la importancia de un pensamiento que comprenda que tanto el conocimiento del todo depende del conocimiento de las partes, como el conocimiento de las partes depende del conocimiento del todo. Reconoce que es necesario sustituir un pensamiento que aísla y separa por un pensamiento que distingue y une y entiende que es necesario sustituir el pensamiento disyuntivo y reductor por un pensamiento complejo, en el sentido original del término complexus: que es tejido en conjunto ${ }^{(20)}$.

Así, el cuidar/cuidado de enfermería, en la actualidad, sólo puede entenderse a la luz del pensamiento complejo, cuya comprensión requiere mirar las múltiples dimensiones que lo componen. La construcción del cuidar/cuidado de enfermería complejo implica, por lo tanto, considerar la articulación de conocimientos que trascienden la linealidad del hacer y que implican un cierto grado de desorden e incertidumbre. Este proceso, a primera vista contradictorio, es esencial para superar la lógica de la fragmentación, de la previsibilidad y del excesivo orden - recetas listas de cuidar/cuidado - aún muy arraigado en el ser y el hacer de enfermería.

Pensar el cuidar/cuidado de enfermería a la luz del referencial de la complejidad, se remonta a una tradición histórico-hegemónica, marcada por las relaciones verticalizadas y predecibles integradas y en el que predominan las relaciones sujeto objeto. En éstas, el conocimiento de enfermería estaba acuñada en un cierto grado de superioridad, como siendo más importante que del usuario. Así, para ser competente, profesionalmente, especialmente en el enfoque técnico, era necesario tener una respuesta preparada para posibles preguntas y/o situaciones de la vida cotidiana.Se sabe, sin embargo, que el cuidar/cuidado de enfermería no es lineal y predecible, lo que significa que este no puede reducirse a acciones específicas, ser dividido en partes más simples o incluso estar en posesión de la certeza absoluta y predecible para las diferentes realidades y situaciones. El cuidar/cuidado como un fenómeno complejo debe ser aprehendido como un todo, y este todo es mayor que la suma de las partes. Mientras el sistema de cuidado en la lógica cartesiana se proyectaba a partir de una receta lógica de preguntas y respuestas, el cuidado complejo se procesa de forma circular, interactiva y asociativa, a través de auto-organización continua y permanente $^{(21)}$.

En este sentido, quedar "sin respuesta y sin lo que pensar, decir o hacer" es algo comprensible y aceptable, ya que el cuidar/cuidado complejo comporta incertidumbres, inestabilidades e imprevisibilidades. Significa decir, que ningún profesional de enfermería no necesita desesperarse, angustiarse o deprimirse ante las situaciones adversas para las cuales no encuentran respuestas objetivas. Es importante que los profesionales a través del cuidado complejo, exploren creativamente sus propios futuros, mientras se adaptan y evolucionan dentro de un ambiente al cual son internamente sensibles y adaptables. Es esencial que los enfermeros, independientemente de su formación profesional, se dejen perturbar y volverse inestable por el referencial de la complejidad, con el fin de romper los paradigmas tradicionales y disolver patrones rígidos de pensamiento o conducta, basada en la linealidad de acciones específicas de cuidar/cuidado. 
Al contrario de un simple sistema, el cuidar/cuidado de enfermería como un fenómeno complejo tiene muchas partes/dimensiones que interactúan entre sí. En este proceso sistémico, en el que las diferentes partes/dimensiones constituyen la totalidad, el movimiento es generado y dinamizado por la forma en que éstos interactúan. Por lo tanto, cuanto más intensas sean las relaciones, interacciones y asociaciones que procesan el cuidar/cuidado, mayores serán las posibilidades de diálogo creativo y mayor el crecimiento y la calidad de la atención. Así, la necesidad de superar la lógica de la fragmentación, la previsibilidad y del excesivo orden - recetas listas de cuidar/cuidado, debe gradualmente ser asumida como actitud proactiva y emancipadora por los profesionales de enfermería. La aprehensión del cuidado como un fenómeno complejo es, por tanto, fundamental para inducir el nuevo, el creativo y posibilitar relaciones próximas, dialógicas y humanizada.

\section{REFERENCIAS}

1. Gepeses. Grupo de Estudos e Pesquisa em Empreendedorismo Social da Enfermagem e Saúde. Validação de conceito de viver saudável à luz da complexidade. Conceito discutido e validado no grupo de pesquisa; 2011.

2. Gepeses. Grupo de Estudos e Pesquisa em Empreendedorismo Social da Enfermagem e Saúde. Validação de conceito de enfermagem à luz da complexidade. Conceito discutido e validado no grupo de pesquisa; 2011.

3. Backes DS. Empreendedorismo social da enfermagem: rupturas e avanços. Santa Maria: Centro Universitário Franciscano; 2012.

4. Backes DS, Backes MS, Erdmann AL. [A prática social sistêmica do enfermeiro na perspectiva luhmanniana]. Rev. esc. enferm. USP, 2011; 45(1):116-121.

5. Backes DS, Erdmann AL, Büscher A. [Evidenciando o cuidado de enfermagem como prática social]. Rev Latino-am Enferm. 2009; 17(6):988-994.

6. Backes DS. Vislumbrando o cuidado de enfermagem como prática social empreendedora [tese]. Florianópolis: Universidade Federal de Santa Catarina; 2008.

7. Büscher A. Negotiating helpful action: a substantive theory on the relationship between formal and informal care [thesis]. Finland: University of Tampere, Department of Nursing Science; 2007.

8. Erdmann AL, Sousa FGM, Backes DS, Mello ALSF. [Construindo um modelo de sistema de cuidados]. Acta Paul Enferm. 2007; 20(2):180-185.

9. Backes DS, Marinho MR, Costenaro RS, Nunes, S, Rupolo I. [Repensando o ser enfermeiro docente na perspectiva do pensamento complexo]. Rev Brasil Enferm. 2010; 63(3):421-426.

10. Falcón GCS, Erdmann AL, Backes DS. [Significados do cuidar na promoção da saúde]. Rev Latino-am Enferm. 2008; 16(3):419-424.

11. Sales CA, Violin MR, Waidman MAP, Marcon SS, Silva MAP. [Emotions of people living with ostomies: existencial comprehension]. Rev Esc Enferm USP 2010; 44(1):221-228.

12. Gepeses. Grupo de Estudos e Pesquisa em Empreendedorismo Social da Enfermagem e Saúde. Validação de conceito de cuidado de enfermagem à luz da complexidade. Conceito discutido e validado no grupo de pesquisa; 2011.

13. Morin E. Ciência com consciência. 10를 ed. Rio de Janeiro: Bertrand Brasil; 2007.

14. Morin E. Introdução ao conhecimento complexo. 4a $\mathrm{Ed}$. Porto Alegre: Editora Moridional Ltda; 2011.

15. Morin E. Complexidade e ética da solidariedade. $3^{\mathrm{a}}$ ed. Porto Alegre: Sulina; 2002. 
16. Morin E, Prigogine I. A sociedade em busca de valores: para fugir á alternativa entre o cepticismo e o dogmatismo. Lisboa: Instituto Piaget; 2003.

17. Morin E. Ciência com consciência. 14aㅡ ed. Rio de Janeiro: Bertrand Brasil; 2010.

18. Backes DS, Erdmann AL, Büscher A. [Demonstrating nursing care as a social practice]. Rev Latino-am. Enferm. 2008; 17(3):988-994.

19. Morin E. Introdução ao pensamento complexo. $6^{\underline{a}}$ ed. Lisboa: Stória Editores; 2011.

20. Morin E. A cabeça bem-feita: repensar a reforma, reformar o pensamento. $17^{\mathrm{a}} \mathrm{ed}$. Rio de Janeiro (RJ): Bertrand Brasil; 2010.

21. Menossi MJ, Lima RAG, Corrêa AK. [A dor e o desafio da interdisciplinaridade no cuidado à criança]. Rev Latino-am Enferm. 2008; 16(3):489-494. 